\title{
Signal manipulation through horn antennas loaded with metamaterial-inspired particles: A review
}

\author{
Mirko Barbuto ${ }^{1, *}$, Davide Ramaccia ${ }^{2}$, Fabrizio Trotta $^{3}$, Filiberto Bilotti ${ }^{2}$, and Alessandro Toscano ${ }^{2}$ \\ 1 Niccolò Cusano University, 00166 Rome, Italy \\ 2 Department of Engineering, RomaTre University, 00146 Rome, Italy \\ 3 Antenna Department, Elettronica S.p.A., 00131 Rome, Italy
}

Received 26 August 2015 / Accepted 23 October 2015

\begin{abstract}
This review paper summarizes our latest results on designing metamaterial-inspired particles that can be inserted inside horn antennas to provide signal manipulation capabilities to the overall system. Metamaterial-inspired band-pass filtering modules, based on the use of either bi-omega particles or complementary electrically small resonators, are firstly presented. The bi-omega particle enables the antenna to perform filtering operations on the received signal to radiate/receive only the narrowband signal of interest, improving, thus, the signal-to-noise ratio. The second module, besides filtering the unwanted signals out, allows converting the linearly polarized mode of the feeding waveguide into a circular polarized field in the horn, and vice-versa. Moreover, some band-stop filters, based on the employment of single or coupled metamaterial-inspired resonators, are presented. All these components are of extremely compact size and, therefore, can be easily realized with standard PCB technology and added to standard horn antennas to increase their functionalities.
\end{abstract}

Key words: Filtering antennas, Horn antennas, Metamaterial-inspired particles, Signal manipulation.

\section{Introduction}

Artificial electromagnetic materials, metamaterials, and metasurfaces have revolutionized the design of microwave and optical components, allowing to obtain better performances with respect to conventional solutions [1-8] or novel functionalities [9-14]. In particular, in the last years, there was a strong interest in the use of metamaterials for performing the manipulation of the electromagnetic waves. In the past, such ability has been achieved by using dielectric lenses, frequency selective surfaces and polarizers, but the additional degrees of freedom enabled by properly engineered metamaterials allows performing novel operation directly on the electromagnetic waves passing through them. For instance, the design flexibility of composite-right-left-handed transmission lines has been used in [15] for designing different signal processing systems, such as delay lines, pulse generators, and pulseposition modulators. Moreover, it has been shown that an array of waveguides, working at their cut-off frequency for emulating an epsilon-near-zero metamaterial $[16,17]$, can be used to perform a Fourier Transform of the electromagnetic signal propagating through them [18]. Finally, it has been proven that to perform some mathematical operations, such as convolution,

*e-mail: mirko.barbuto@unicusano.it integration and spatial differentiation, on an electromagnetic wave a metamaterial slab that is only one wavelength thick can be used [19].

However, the intrinsic frequency dispersion of 3D bulky metamaterials and the required engineered spatial dispersion makes the aforementioned solutions rather difficult to be realized. Moreover, in many cases losses are so high that the amplitude of the electromagnetic signal becomes impractical. In this framework, the authors have recently proposed to only use a single metamaterial-inspired electrically-small particle to perform signal manipulation. The advantage of such an approach is twofold: (i) the electromagnetic response of the signal manipulator overlaps the one of the metamaterialinspired particle, and, thus, it can be easily predicted through an equivalent circuit model [20-22], (ii) being the particle electrically small, it can be easily integrated in conventional components for giving them new features.

Among the several signal manipulations that can be performed on an electromagnetic wave, we focus our attention on the filtering ones, which are typically performed by the front-end of a receiving system. In fact, any standard communication system employs filtering modules for removing the unwanted frequency components from the received signal. For instance, a band-rejection filter can be inserted in the 


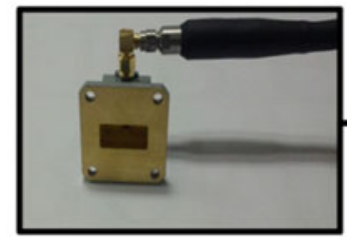

Coaxial to waveguide transition

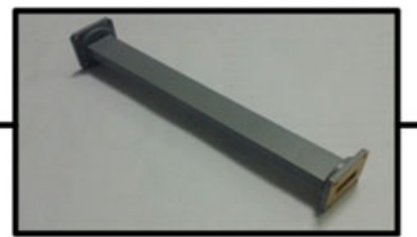

Regular rectangular waveguide

Figure 1. Schematic block diagram of the proposed system.

front-end of the receiver to suppress interfering signals of other services operating within the same frequency band of the antenna. On the contrary, a band-pass filter can be used for reducing the background noise. Therefore, regardless of the type of the filter, the signal-to-noise ratio (SNR) significantly increases, improving the overall performances of the receiving antenna. In this framework, the possibility to have a highly efficient manipulation of the electromagnetic signal by using an extremely compact structure would represent an important achievement.

Although the integration of the antenna and the filters on a single microwave component has been proposed for planar printed antennas [23-26], where it is easy to integrate a conventional LC circuits or resonant particles acting as filters, little efforts have been made to embed filtering module directly inside horn antennas. Some attempts can be found in [27-29] based on the use of metal or dielectric posts or substrate integrated waveguide cavity frequency selective surface. However, they work only for one polarization and, due to their complex structure, need to be realized together with the antenna. On the other hand, the proposed metamaterial-inspired particles for signal manipulation can be easily integrated in any existing antenna system, as shown in the schematic block diagram of Figure 1. In particular, the integrated filter element, consisting of a single particle, is inserted between a regular waveguide and the horn antenna, which easily gains the filtering feature. This choice allows also keeping the system very compact and ensures the backward compatibility with the pre-existing communication systems. Moreover, the use of metamaterialinspired particle can also add other advanced functionalities to the overall system such as polarization-control capabilities and non-reciprocity [30, 31].

In this paper, we review our recent work on metamaterialinspired filtering modules that can be used in both transmitting and receiving self-filtering antennas [32-37]. In particular, we review a band-pass filtering module for linear polarization [34], a linear-to-circular polarization transformer with a band-pass behavior [35], and some notched-band filters $[36,37]$ that, consisting of compact resonant inclusions, can be easily integrated in horn antennas. Moreover, we show here how the use of different metamaterial-inspired particles allows performing different operations on the electromagnetic signals.

\section{Band-pass filtering modules}

Let us start by considering the band-pass feature of the proposed metamaterial-inspired particles. As discussed in

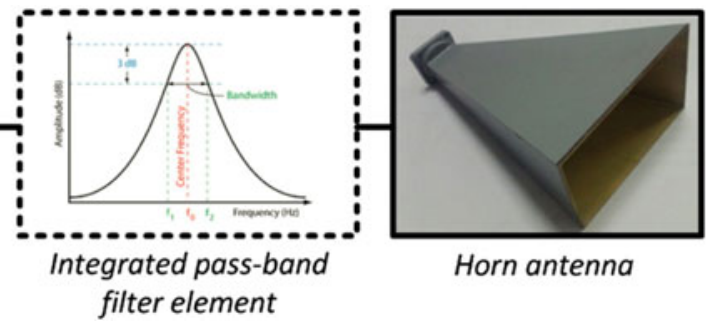

filter element

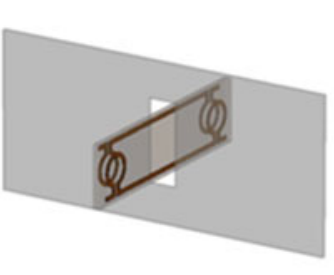

(a)

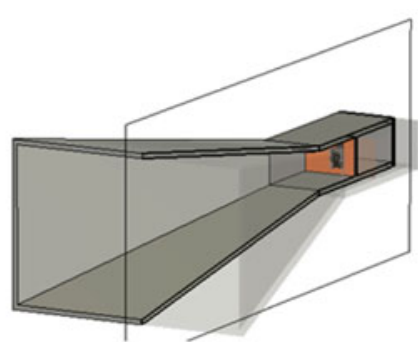

(b)

Figure 2. Geometrical sketch of the band-pass filtering module working for linear polarization (a) and of the overall structure consisting of the filtering module inserted between a WR-62 waveguide connected to a pyramidal horn antenna (b).

Section 1, in a standard communication system employing horn antennas, a regular horn is connected to a feeding waveguide that is excited by a proper coaxial-to-waveguide transition. As it is well known, these components can support the propagation and radiation of electromagnetic signals over a broad frequency range. However, the signal to be received is typically band-limited (especially in satellite communications) and a band-pass filter can be used for selecting the band of interest and rejecting the out-of-band background noise. In the following, we present two metamaterial-inspired particles, which work for linear polarization $(L P)$ and circular polarization $(C P)$, respectively, comparing their performances.

\subsection{Linear polarization}

The first proposed particle, shown in Figure 2a, consists of a connected bi-omega particle placed through an aperture drilled in a metallic screen. In particular, the structure consists of two identical metallizations printed with opposite orientations on the two sides of a dielectric slab (GML 2032). Each metallizations can be seen as two individual bi-omega resonators connected through two metallic strips. The operating principle of such a particle as pass-band filter is based on the ability of the two identical bi-omega inclusions on the opposite side of the metallic screen to act as receiving and radiating electrically small antennas. At the resonant frequency, the energy captured by the first bi-omega from the $\mathrm{TE}_{10}$ propagating mode of the waveguide is transferred through the two metallic wires to the second bi-omega that, in turn, radiates 


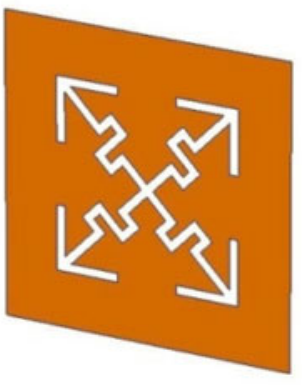

(a)

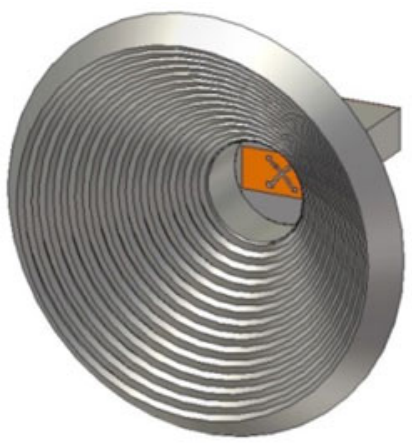

(b)
Figure 3. Geometrical sketch of the band-pass filtering module working for circular polarization (a) and of the overall structure consisting of the filtering module inserted between a WR-62 waveguide connected to a corrugated conical horn antenna (b).

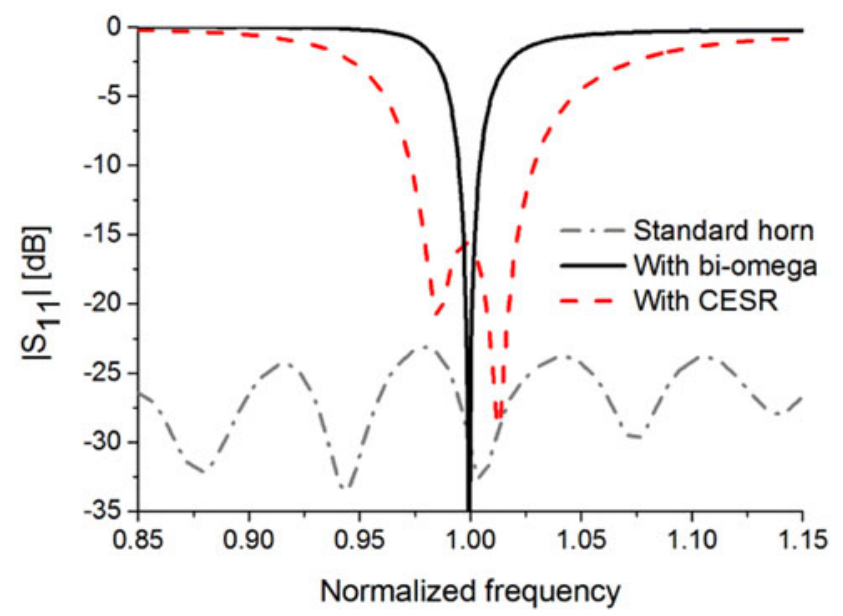

Figure 4. Reflection coefficient magnitude of a standard horn antenna (gray dash-dotted line) and of the same horn with a biomega particle (black solid line) or a CESR (red dashed line).

and excites the same mode on the other side of the screen. It is now easy to understand that, if the particle is placed between the horn and the rectangular waveguide (as shown in Figure $2 \mathrm{~b}$ ), the antenna can perform a band-pass filtering process on the received signal.

In order to verify its effectiveness, the simulated reflection coefficient of the overall structure at the input port as a function of the normalized frequency is reported in Figure 4 (black solid line). It shows that a good impedance matching is achieved only in a narrow frequency band around the normalized resonant frequency, proving that the signal is not reflected back in that frequency band. In order to show that the energy is not absorbed by the particle, we report also the normalized realized gain in the main beam direction (Figure 5 - black solid line), which presents the same values of a regular horn antenna around the band-pass range and rapidly decreases moving away from it, confirming the efficient energy transfer and low loss introduced by the particle.

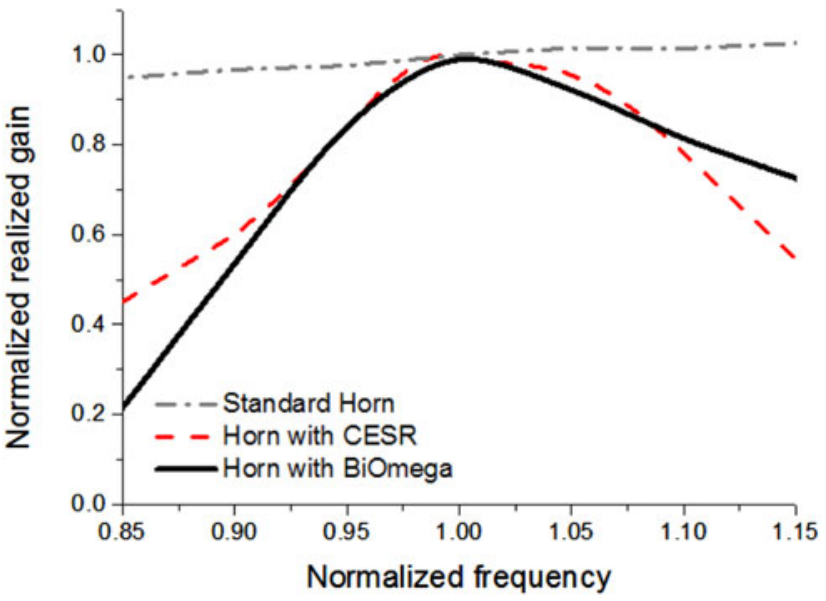

Figure 5. Normalized realized gain of a standard horn antenna (gray dash-dotted line) and of the same horn with a bi-omega particle (black solid line) or a CESR (red dashed line).

\subsection{Circular polarization}

The second proposed particle has been designed to operate on circular polarized signals. Such a particle needs to be properly designed because it is necessary that at least one symmetry plane orthogonal to the transverse section of the waveguide is present in order to radiate effectively both orthogonal components of the $\mathrm{CP}$ field. The connected biomega particle fails in this context because it is excited mainly by the magnetic component of the $\mathrm{TE}_{10}$ propagating mode in the rectangular waveguide, which has to be orthogonal to the bi-omega plane.

To this end, we have replaced the bi-omega particle with a Complementary Electrically Small Resonator (CESR), shown in Figure 3a, which has been inserted at the junction between a rectangular waveguide and a corrugated conical horn, as shown in Figure 3b. Properly designing the particle, we can convert the linear polarization of the $\mathrm{TE}_{10}$ mode in the waveguide into a circular one in the radiated field and vice-versa, in addition to filtering the received signal. In particular, the proposed device consists of two mutually orthogonal meander-line slot dipoles that, in order to obtain a circular polarization operation, are terminated with arrows that have slightly different dimensions. The filtering behavior is confirmed by the reflection coefficient and the realized gain of the overall structure, reported in Figures 4 and 5 (red dashed lines), respectively, as a function of the normalized frequency. The radiation of a circular polarized field, instead, is proven by the axial ratio, shown in Figure 6, which is below $3 \mathrm{~dB}$ around the resonant frequency of the particle.

It is worth noticing that the common feature of the two proposed filtering modules for LP and CP fields is the use of a metallic screen that does not allow the propagation of the electromagnetic field, except in a narrow frequency range around the resonant frequency of the employed particle. As it will be discussed in the following section, this approach cannot be employed for designing band-stop filters that, in contrast, allow passing most frequencies unaltered while strongly attenuate those in a narrow frequency range. 


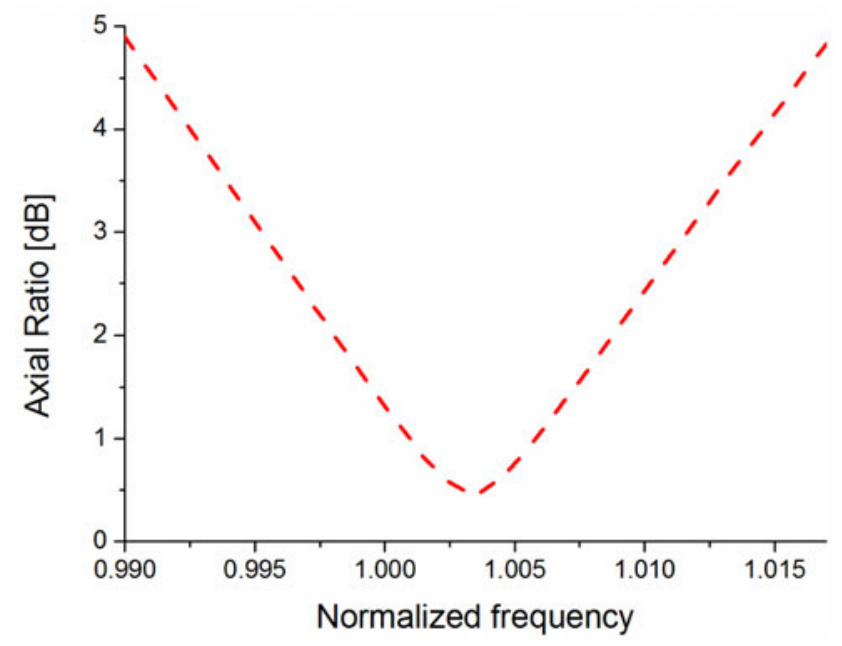

Figure 6. Axial ratio in the main beam direction of a horn antenna loaded with a CESR.

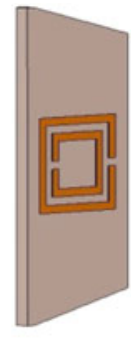

(a)

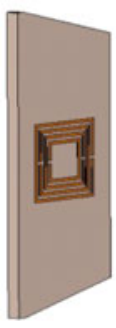

(b)

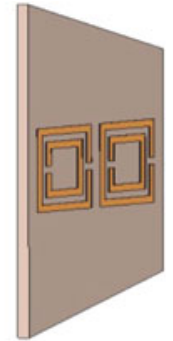

(c)

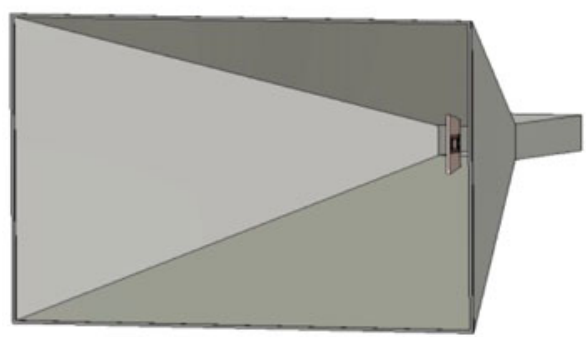

(d)

Figure 7. Geometrical sketch of the band-stop filtering modules consisting of a single SRR (a), a single MSRR (b), two coupledSRRs (c) and of the overall structure consisting of the filtering module inserted between a WR-90 waveguide connected to a pyramidal horn antenna $(d)$.

\section{Band-stop filtering modules}

In this section, we present some particles that can be used for removing a narrowband interfering signal from the receiving spectrum of a horn antenna. A very simple method consists in using a single Split-Ring Resonator (SRR) placed directly inside the throat of a pyramidal horn antenna, as shown in Figure 7. Excited by the electromagnetic field in the horn, the SRR resonates and generates strong mismatch and loss phenomena around its resonant frequency, which can be predicted by equivalent circuit models [20,21]. Please, note that,

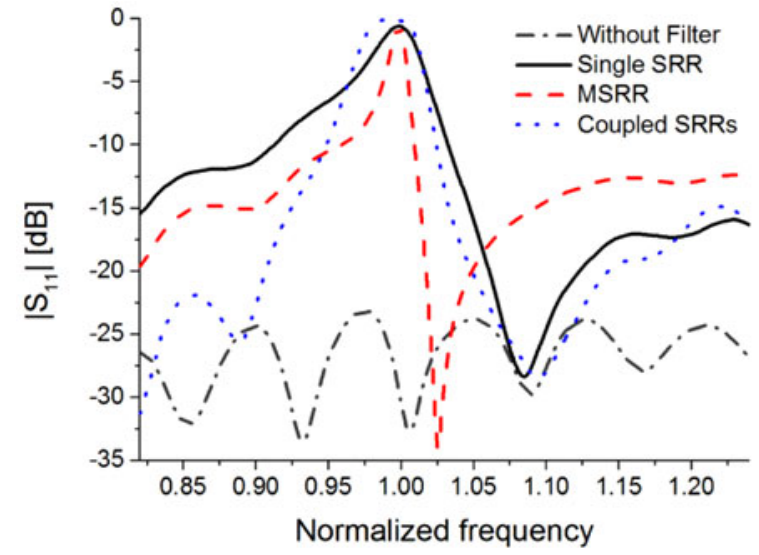

Figure 8. Reflection coefficient magnitude of a standard horn antenna (gray dash-dotted line) and of the same horn with a single SRR (black solid line), a MSRR (red dashed line) or two coupled SRRs (blue dotted line).

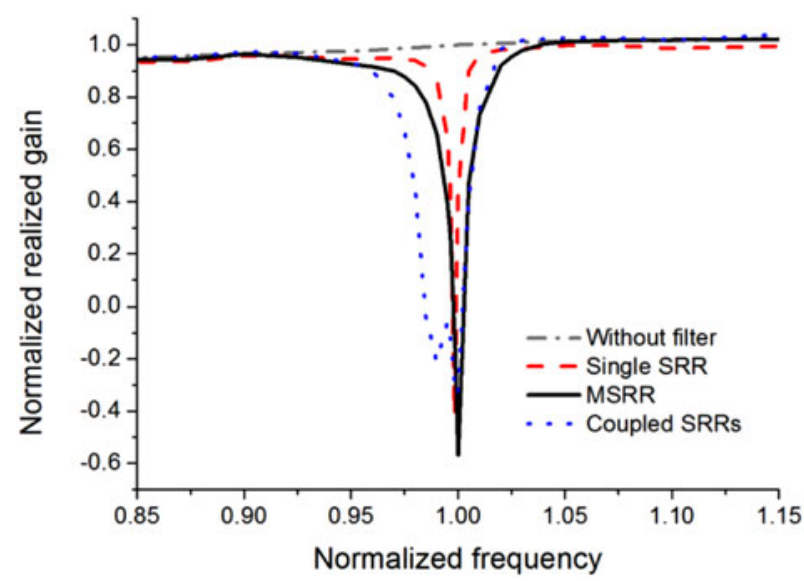

Figure 9. Normalized realized gain of a standard horn antenna (gray dash-dotted line) and of the same horn with a single SRR (black solid line), a MSRR (red dashed line) or two coupled SRRs (blue dotted line).

in order to have maximum interaction with the field, the SRR has to be parallel to the E-plane of the horn. In this way, the electric field will be parallel to the gaps of the SRR and the magnetic field will be normal to its plane, inducing the maximum possible currents on the metallic strips of the SRR. In contrast, moving away from its resonant frequency, the SRR does not interact anymore with the electromagnetic signal that is allowed to pass unaltered. This behavior is confirmed both by the reflection coefficient and the realized gain of the overall structure reported in Figures 8 and 9, respectively.

Moreover, by using the same approach, different metamaterial-inspired particles can be used. In fact, each of these has a different Q-factor and, thus, different frequency response in terms of fractional bandwidth. In particular, here we report the comparison between the SRR and a multiple-SRR (MSRR) or two coupled SRRs (C-SRRs), which are able to reduce or broaden the rejected band, respectively. The first one exhibits a higher Q-factor, due to its electrically smaller dimensions 
with respect to the conventional SRR, whereas the critical coupling between two properly spaced SRRs can be exploited for achieving a larger bandwidth compared to the one of a single resonator [37]. Therefore, as shown in Figures 8 and 9, we are able to obtain a different operation bandwidth depending on the used inclusion. In this way, the proposed approach can be employed in several systems characterized by different requirements in term of rejection bandwidth.

\section{Conclusion}

In this contribution, we have reviewed and discussed our recent results about signal manipulation process obtained through horn antennas loaded with metamaterial-inspired particles. In particular, we have shown that, depending on the used inclusion, a wideband horn antenna can be enabled to perform different manipulations on the transmitted and received signals, such as band-pass and band-stop filtering processes, and polarization transformation. Therefore, the proposed modules can be considered as a new family of microwave components that perform signal manipulation directly inside the radiating element of a communication system. By using these compact modules, we reduce the number of components constituting a communication system, with clear advantages in terms of cost, weight, complexity, and space occupancy.

Please, note that all the reported results have been obtained by using the full-wave simulator CST Microwave Studio [38]. In order to further confirm their effectiveness, most of the designed modules have been also manufactured and characterized both in terms of impedance matching and radiation performance. For the sake of brevity we have not reported the corresponding results, presented in [34-37], but we remark here that they are in good agreement with the simulated ones and confirm the different signal manipulation achievable with the proposed modules.

\section{References}

1. N. Engheta, R.W. Ziolkowski (Eds.), Metamaterials: physics and engineering explorations, Wiley-IEEE Press, Hoboken, NJ, 2006.

2. T.J. Cui, R. Liu, D.R. Smith, Metamaterials: theory, design, and applications, Springer Science, New York, 2010.

3. F. Bilotti, L. Sevgi, Metamaterials: definitions, properties, applications, and FDTD-based modeling and simulation, International Journal of RF and Microwave Computer-Aided Engineering 22 (2012) 422-438.

4. Y. Yang, B. Zhang, E. Li, H. Chen, Towards omnidirectional, large scale, full polarization, and broadband practical invisibility cloaks: challenges and progress, EPJ Applied Metamaterials 1 (2014) 7.

5. D. Ramaccia, A. Toscano, A. Colasante, G. Bellaveglia, R. Lo Forti, Inductive tri-band double element FSS for space applications, Progress in Electromagnetics Research C 18 (2011) 87-101.

6. D. Ramaccia, F. Bilotti, A. Toscano, L. Vegni, Dielectric-free multi-band frequency selective surface for antenna applications,
COMPEL: The International Journal for Computation and Mathematics in Electrical and Electronic Engineering 32 (2013) 1868-1875.

7. D. Ramaccia, F. Scattone, F. Bilotti, A. Toscano, Broadband compact horn antennas by using EPS-ENZ metamaterial lens, IEEE Transactions on Antennas and Propagation 61 (2013) 2929-2937.

8. D. Ramaccia, A. Toscano, F. Bilotti, A new accurate model of high-impedance surfaces consisting of circular patches, Progress in Electromagnetics Research M 21 (2011) 1-17.

9. M. Barbuto, A. Monti, F. Bilotti, A. Toscano, Design of a nonFoster actively loaded SRR and application in metamaterialinspired components, IEEE Transactions on Antennas and Propagation 61 (2013) 1219-1227.

10. M. Barbuto, A. Toscano, F. Bilotti, Linear-to-circular polarization transformer using electrically small antennas, in Proc. IEEE APSURSI Int. Symp., 2012, pp. 1-2.

11. D. Ramaccia, F. Bilotti, A. Toscano, Analytical model of a metasurface consisting of a regular array of sub-wavelength circular holes in a metal sheet, Progress in Electromagnetics Research M 18 (2011) 209-219.

12. C. Zhao, J. Zhang, Y. Liu, Light manipulation with encoded plasmonic nanostructures, EPJ Applied Metamaterials 1 (2014) 6.

13. M. Barbuto, F. Bilotti, A. Toscano, Design of a multi-functional SRR-loaded printed monopole antenna, International Journal of $\mathrm{RF}$ and Microwave Computer-Aided Engineering 22 (2012) 552-557.

14. I. Semchenko, S. Khakhomov, A. Balmakou, S. Tretyakov, The potential energy of non-resonant optimal bianisotropic particles in an electromagnetic field does not depend on time, EPJ Applied Metamaterials 1 (2014) 4.

15. S. Gupta, C. Caloz, Analog signal processing in transmission line metamaterial structures, Radioengineering 18 (2009) 155-167.

16. W. Rotman, Plasma simulation by artificial dielectrics and parallel-plate media, IRE Transactions on Antennas and Propagation 10 (1962) 82-95.

17. B. Edwards, A. Alù, M.G. Silveirinha, N. Engheta, Reflectionless sharp bends and corners in waveguides using epsilonnear-zero effects, Journal of Applied Physics 105 (2009) 044905.

18. M. Navarro-Cia, M. Beruete, M. Sorolla, N. Engheta, Lensing system and Fourier transformation using epsilon-near-zero metamaterials, Physical Review B 86 (2012) 165130-165136.

19. A. Silva, F. Monticone, G. Castaldi, V. Galdi, A. Alù, N. Engheta, Performing mathematical operations with metamaterials, Science 343 (2014) 160-163.

20. F. Bilotti, A. Toscano, L. Vegni, K. Aydin, K. Boratay, E. Ozbay, Equivalent circuit model for the design of metamaterials based on artificial magnetic inclusions, IEEE Transactions Microwave Theory and Technique 55 (2007) 2865-2873.

21. D. Ramaccia, L. Di Palma, D. Ates, E. Ozbay, A. Toscano, F. Bilotti, Analytical model of connected bi-omega: robust particle for the selective power transmission through subwavelength apertures, IEEE Transactions on Antennas and Propagation 62 (2014) 2093-2101.

22. M. Barbuto, A. Alù, F. Bilotti, A. Toscano, L. Vegni, Characteristic impedance of a microstrip line with a dielectric overlay, COMPEL - The International Journal for Computation and Mathematics in Electrical and Electronic Engineering 32 (2013) 1855-1867. 
23. C. Yu, W. Hong, Z. Kuai, H. Wang, Ku-band linearly polarized omnidirectional planar filtenna, IEEE Antennas and Wireless Propagation Letters 11 (2012) 310-313.

24. X. Chen, F. Zhao, L. Yan, W. Zhang, A compact filtering antenna with flat gain response within the passband, IEEE Antennas and Wireless Propagation Letters 12 (2013) 857-860.

25. M. Naser-Moghadasi, R.A. Sadeghzadeh, T. Sedghi, T. Aribi, B.S. Virdee, UWB CPW-fed fractal patch antenna with band-notched function employing folded t-shaped element, IEEE Antennas and Wireless Propagation Letters 12 (2013) 504-507.

26. J.Y. Siddiqui, C. Saha, Y.M.M. Antar, Compact SRR loaded UWB circular monopole antenna with frequency notch characteristics, IEEE Transactions on Antennas and Propagation 62 (2014) 4015-4020.

27. B. Froppier, Y. Mahe, E.M. Cruz, S. Toutain, Design of a filtering antenna: application to the electromagnetic horn, IEEE AP-S Symposium (Digest) 2 (2003) 1173-1176.

28. W.G. Yeo, T.Y. Seo, J.W. Lee, C.S. Cho, H-plane sectoral filtering horn antenna in PCB substrates using via fences at millimeterwave, in Proc. 37th Eur. Microw. Conf., Munich, Germany, 2007, pp. 818-821.

29. G.Q. Luo, W. Hong, H.J. Tang, J.X. Chen, X.X. Yin, Z.Q. Kuai, $\mathrm{K}$. $\mathrm{Wu}$, Filtenna consisting of horn antenna and substrate integrated waveguide cavity FSS, IEEE Transactions on Antennas and Propagation 55 (2007) 92-98.

30. M. Barbuto, F. Bilotti, A. Toscano, Design of a circular polarized horn filtenna using complementary electrically small resonators, in Proc. of Metamaterials'2013, Bordeaux, FR, 2013, pp. 91-93.
31. D. Ramaccia, F. Bilotti, A. Toscano, Angular Momentumbiased metamaterials for filtering waveguide components and antennas with non-reciprocal behavior, in Proc. of Metamaterials'2014, Copenhagen, DK, 2014, pp. 250-252.

32. D. Ramaccia, L. Di Palma, G. Guarnieri, S. Scafè, A. Toscano, F. Bilotti, Balanced and unbalanced waveguide power splitters based on connected bi-omega particles, Electronics Letters 49 (2013) 1504-1506.

33. M. Barbuto, F. Bilotti, A. Toscano, Novel waveguide components based on complementary electrically small resonators, Photonics and Nanostructures 12 (2014) 284-290.

34. F. Bilotti, L. Di Palma, D. Ramaccia, A. Toscano, Self-filtering low-noise horn antenna for satellite applications, IEEE Antennas and Wireless Propagation Letters 11 (2012) 354-357.

35. M. Barbuto, F. Trotta, F. Bilotti, A. Toscano, A combined bandpass filter and polarization transformer for horn antennas, IEEE Antennas and Wireless Propagation Letters 12 (2013) 1065-1068.

36. M. Barbuto, F. Trotta, F. Bilotti, A. Toscano, Horn antennas with integrated notch filters, IEEE Transactions on Antennas and Propagation 63 (2015) 781-785.

37. M. Barbuto, F. Trotta, F. Bilotti, A. Toscano, Varying the operation bandwidth of metamaterial-inspired filtering modules for horn antennas, Progress in Electromagnetics Research C 58 (2015) 61-68.

38. CST Studio Suite 2014, CST Computer Simulation Technology AG, Available at: www.cst.com

Cite this article as: Barbuto M, Ramaccia D, Trotta F, Bilotti F \& Toscano A: Signal manipulation through horn antennas loaded with metamaterial-inspired particles: A review. EPJ Appl. Metamat. 2015, 2, 5. 